\title{
BMJ Open Validation of the health assets index in the Australian inpatient setting: a multicentre prospective cohort protocol study
}

Katherine Gregorevic, ${ }^{1}$ Ruth E Hubbard, ${ }^{2}$ Nancye May Peel, ${ }^{2}$ Wen Kwang Lim $^{3}$

To cite: Gregorevic K, Hubbard RE, Peel NM, et al. Validation of the health assets index in the Australian inpatient setting: a multicentre prospective cohort protocol study. BMJ Open 2018;8:e21135. doi:10.1136/ bmjopen-2017-021135

- Prepublication history for this paper is available online. To view these files, please visit the journal online (http://dx.doi. org/10.1136/bmjopen-2017021135).

Received 22 December 2017 Revised 28 March 2018 Accepted 18 April 2018
Check for updates

${ }^{1}$ Department of Aged Care, Northern Health, Epping, Victoria, Australia

${ }^{2}$ Centre for Research in Geriatric Medicine, The University of Queensland, Brisbane, Queensland, Australia

${ }^{3}$ Department of Medicine and Aged care, Melbourne Health, Parkville, Victoria, Australia

Correspondence to Dr Katherine Gregorevic; kate.gregorevic@gmail.com

\section{ABSTRACT}

Introduction It is well known that frail older adults are at increased risk for mortality and functional decline on admission to hospital. Systematic review demonstrates that health assets are associated with improved outcomes for hospitalised older adults. The health assets index (HAl) has been developed to measure health assets in the hospital setting. A protocol has been developed to determine the predictive validity of the HAI for frail older adults.

Methods and analysis The HAl was developed based on a systematic review and secondary analysis of the interRAI-Acute Care (interRAI-AC) dataset. A pilot study was undertaken to refine the tool. The validation study will be a multicentre prospective cohort. Participants will be adults aged 70 years and older with an unplanned admission to hospital. Frailty, illness severity and demographic data will also be recorded. The primary outcomes are mortality at 28 days postdischarge and functional decline at the time of discharge from hospital. The primary hypothesis is that a higher score on the HAI will mitigate the effects of frailty for hospitalised older adults. The secondary outcomes to be recorded are length of stay, readmission at 28 days and functional status at 28 days postdischarge. The correlation between HAI and frailty will be explored. A multivariate analysis will be undertaken to determine the relationship between the HAI and the outcomes of interest.

Ethics and dissemination Ethical approval has been obtained from Austin Health Human High Risk Ethics Committee. The results will be disseminated in peerreviewed journals and research conferences. This study will determine whether the HAI has predictive validity for mortality and functional decline for hospitalised, frail older adults.

\section{INTRODUCTION}

The health assets index (HAI) (see table 1) was created to capture the cumulative effect of health assets. The aim of this study is to determine whether the HAI has predictive validity in the inpatient setting for older adults. It is proposed that the HAI will improve prognostication when measured concurrently with frailty. Determination of health assets
Strengths and limitations of this study

- This is the first study to systematically measure health assets in the hospital setting.

- Attempts have been made to minimise the burden for unwell participants by including significant amounts of routine data to decrease barriers to participation.

Despite completion of a systematic review, it is possible that not all health assets have been identified.

- The health assets identified may not be applicable to other sociocultural settings.

associated with improved outcomes may also lead to new strategies to improve survival and well-being following hospital admission.

\section{BACKGROUND}

Health assets are protective factors that support health and well-being, rather than risk factors that are associated with disease. ${ }^{1}$ Health assets are a way to operationalise the concept of salutogenesis, which describes an approach focusing on factors that support well-being and health rather than factors that cause disease. ${ }^{2}$ This concept was initially developed in the community setting. In a systematic review, individual health assets have been demonstrated to decrease the risk of adverse outcomes including mortality, functional decline, for residential care, readmission and length of stay for older adults. ${ }^{1}$ Some examples of assets identified included higher level of educational attainment, social engagement, subjective well-being and financial resources. ${ }^{1}$

It has been demonstrated that health deficits can be measured at the time of hospital admission by comprehensive geriatric assessment and used to construct a frailty index, which has a cumulative association with mortality and length of stay. ${ }^{3-5}$ The frailty 
Table 1 Health assets index

\section{Domain and} question number Question

\section{Proposed scoring system}

\begin{tabular}{|c|c|c|}
\hline \multicolumn{3}{|c|}{ Education } \\
\hline 1 & $\begin{array}{l}\text { At approximately what age did you start school? } \\
\text { At approximately what age did you finish school? }\end{array}$ & To be determined depending on spread. \\
\hline \multicolumn{3}{|c|}{ Primary language } \\
\hline 2 & What is your primary language? & Need to determine association. \\
\hline \multicolumn{3}{|c|}{ Carer } \\
\hline 4 & $\begin{array}{l}\text { Do you have a carer or someone you can rely on to help } \\
\text { with day-to-day activities? }\end{array}$ & $\begin{array}{l}0: \text { no. } \\
1: \text { yes. }\end{array}$ \\
\hline 5 & $\begin{array}{l}\text { Do you have a support person who is positive towards } \\
\text { discharge or maintaining residence in the community? }\end{array}$ & $\begin{array}{l}0: \text { no. } \\
1: \text { yes. }\end{array}$ \\
\hline 6 & Do you live alone or with others? & $\begin{array}{l}0: \text { alone. } \\
1: \text { with others. }\end{array}$ \\
\hline \multicolumn{3}{|l|}{ GP } \\
\hline 7 & Do you have a regular GP? & $\begin{array}{l}0: \text { no. } \\
1: \text { yes. }\end{array}$ \\
\hline
\end{tabular}

$\begin{array}{lll}\text { Financial } & \begin{array}{ll}\text { Do you have private health insurance or other form of } \\ \text { health services over such as Department of Veterans' }\end{array} & \begin{array}{l}0: \text { no. } \\ 1: \text { yes. }\end{array} \\ \begin{array}{lll}\text { Affairs Gold Card? } & 0: \text { no. } \\ 9 & \text { Do you own their own home? } & \begin{array}{l}0.5 \text { yes with mortgage. } \\ 1: \text { yes. }\end{array}\end{array}\end{array}$

How do you manage on the income you have available? 0 : it is difficult/impossible most of the time. 0.5 : it is difficult some of the time.

1 : it is mostly/always manageable.

\section{Number of children}

$10 \quad$ How many children do you have?

\section{0: zero.}

0.5 : for one to two.

1: for three or more.

\section{Social engagement}

Can you count on anyone to provide you with emotional 0 : no.

support, for example, talking over a problem, or helping 1: yes.

with a decision?

$\begin{array}{lll}\text { How many times a week do you see or talk to a family } & 0: \text { never. } \\ \text { member or friend who does not live with you? } & 0.5 \text { : less that once a week. } \\ & \begin{array}{ll}\text { In once a week or more. } \\ \text { In the } 3 \text { days prior to the onset of the illness }\end{array} & 0: \text { no days out. } \\ \text { precipitating admission, number of days went out of the } & 0.25: \text { did not go out in last } 3 \text { days but usually } \\ \text { house or building in which he or she resides (no matter } & \text { goes out over a 3-day period. } \\ \text { how short the period). } & 0.5: 1-2 \text { days. } \\ & 1: 3 \text { days. }\end{array}$

\begin{tabular}{|c|c|c|}
\hline \multicolumn{3}{|c|}{ Psychosocial well-being } \\
\hline 15 & Do you have control over the important things in life? & $\begin{array}{l}0: \text { never. } \\
0.5 \text { : sometimes. } \\
\text { 1: mostly. }\end{array}$ \\
\hline 16 & Overall how would you rate your quality of life? & $\begin{array}{l}0: \text { mostly bad. } \\
0.5 \text { : sometimes good, sometimes bad. } \\
\text { 1: mostly good. }\end{array}$ \\
\hline
\end{tabular}


index is a count of deficits across multiple domains including medical, functional, cognitive, psychological and nutritional. Each deficit is given equal weight, and the score is derived by the numerator over the denominator. ${ }^{6}$

Identifying only deficits does not explain why some frail older adults have a good outcome following hospital admission. In the community setting over a period of many years, a higher number of health protective factors decreased the risk of mortality and increased the likelihood of an improvement in health status for frail older adults. ${ }^{7}$ It is yet to be determined whether health assets have a cumulative effect in the hospital setting. As the risk for mortality and functional decline is relatively high, ${ }^{89}$ even a small impact could have a substantial effect at a societal level.

\section{Development of the HAI}

To enable measurement of health assets, the HAI was created. Variables were included based on the systematic review, a secondary analysis of the interRAI dataset ${ }^{3}$ and face validity (see table 1 ).

Variables included will meet the following criteria:

1. Associated with positive health outcomes.

2. Not included in the frailty index.

3. Not present or absent in greater than $95 \%$ of patients.

4. Variables can be binary, continuous or categorical.

5. As a group, the candidate assets must cover a range of domains, for example, social, psychological and socioeconomic.

6. Assets must be age appropriate, for example, being in paid employment is likely to have such low prevalence that it will not provide any meaningful discrimination in older adults.

\section{Scoring of HAl}

The variables will be assigned a score from 0 to 1 .

- Binary variables will be scored as $0=$ assetnot present and $1=$ asset present.

- Categorical variables will be scored according to a range, that is, activity level.

- Continuous and ordinal variables will be transformed into categorical variables by examining spread and judgement; for example, education may be divided as less than 12 years and more than 12 years.

- A higher score will correspond to a higher number of health assets.

\section{OBJECTIVES}

- Determine the distribution of the HAI in hospitalised older adults.

- Examine inter-rater reliability of the HAI.

- Measure the presence of health assets in relation to the presence of frailty.

- Determine whether a higher score on the HAI decreases the chance of mortality for hospitalised older adults.

- Determine whether the HAI decreases the change of hospital-associated functional decline.
- Determine impact of health assets on functional recovery and mortality at 12 months after follow-up.

\section{Primary hypothesis}

- The distribution of score on the HAI index will be related to the frailty score.

- A higher score on the HAI will mitigate the effect of frailty on hospitalised older adults and lead to decreased mortality and functional decline.

\section{Secondary hypothesis}

- A higher score on the HAI will mitigate the effect of frailty on hospitalised older adults and lead to decreased length of stay and readmission.

\section{Study design}

The study protocol has been developed in accordance with the Transparent Reporting of a multivariable prediction model for Individual Prognosis Or Diagnosis (TRIPOD) checklist. ${ }^{10}$ The study is a prospective, observational, multicentre cohort study that will take place in the inpatient hospital setting.

\section{Participants}

\section{Inclusion criteria}

Hospital inpatients who are aged 70 years and above who have an unplanned admission.

\section{Exclusion criteria}

Participants were excluded if they did not speak English or if they had cognitive impairment and no next of kin was available to consent for them.

\section{Predictors}

Predictors included in the study include age, gender, frailty (measured by frailty index) ${ }^{3}$ and modified early warning score ${ }^{11}$ to indicate illness severity. These will be measured at the time of admission to hospital from medical records, participants and next of kin.

\section{Sample size}

The interRAI dataset, which examined a comparable population, had a 28-day mortality of $7.5 \% .^{5}$ Functional decline in Activities of Daily Living (ADLs) and Instrumental Activities of Daily Living (iADLs) was estimated at $35 \%$ based on prior studies in comparable populations. ${ }^{812}$ The CI was set at $95 \%$, and the confidence level was set at 0.05 . This gave the following size:

1. Thirty-day mortality: 134 .

2. Functional status for instrumental ADLs and DADLs 30 days after discharge: 350 .

To account for $10 \%$ loss to follow-up, the planned sample size is 385 .

\section{Consent}

The researcher will speak to the clinical staff to determine whether there are any concerns regarding the patient's cognition and capacity to consent. If any clinical staff raise concerns or if in the subjective judgement of 
the researcher there are concerns, consent will also be obtained from the next of kin or responsible person.

\section{Study procedure}

The participants will be approached by the researcher either on the acute ward or in the emergency department once they have been accepted for admission. The researcher will complete a frailty index and the HAI based on information from the patient, carers and staff. The researchers will administer the HAI twice to a subset of patients to determine inter-rater reliability. The researcher will obtain information regarding illness severity from the medical records, which will be used to inform the Modified Early Warning Score (MEWS). Age and demographic data will also be obtained from medical records. Demographic data will include gender, age, usual place of residence and previous home help services.

Participants will be asked for a contact phone number for follow-up. Participants who are able to consent for themselves will be asked to nominate whether they prefer to be contacted or for the researchers to contact a relative or carer. For participants who were not able to consent, where appropriate, the person responsible will be contacted for follow-up at 30 days after discharge from the hospital.

\section{Primary outcome measures}

1. Mortality during inpatient admission, which will be determined from hospital records.

2. Mortality within 30 days of hospital discharge, which will be determined from hospital records and the registry of births, deaths and marriages.

3. Functional status at the time of hospital discharge measured by Katz activities of daily living, which will be recorded from medical notes, or the need for subacute care or new admission to residential aged care.

4. Functional status at 30 days after hospital discharge that will be obtained by follow-up phone call or examination of medical records, which will be measured by Katz activities of daily living and instrumental activities of daily living or new need for residential care.

\section{Secondary outcome measures}

1. Length of stay, which will be determined from medical records.

2. Readmission, which will be determined from medical records and phone call.

\section{Data management}

Each researcher will be responsible for entering deidentified data into a centralised, password-protected database on RedCap. RedCap is a secure web application for building and managing online surveys and databases, which enables data from all sites to be securely managed on a single database.

\section{Statistical analysis}

Descriptive statistics will be used to examine baseline characteristics including MEWS, frailty index, age, gender and usual place of residence.

The distribution of the individual components will be examined in the population. The distribution of the total score of the HAI will also be examined in the population.

Inter-rater reliability will be performed using Spearman's correlation.

The HAI will be examined in a multivariate, regression model that will include frailty, age, MEWS and gender. Participants who had a score of 0 for ADLs at the time of admission will not be included in the analysis for functional decline due to a floor effect. A negative binomial regression will be used for length of stay to account for skew of data.

\section{ETHICS AND DISSEMINATION}

Research governance has also been obtained for participating sites. The findings of this study will be presented at conferences and disseminated through publication in a peer-reviewed journal.

A pilot study of the HAI was undertaken as a substudy of the interRAI acute care assessment. This pilot study determined that HAI was acceptable to participants.

\section{PATIENT INVOLVEMENT}

Patients were not involved in the development of the study. The results will be published in a peer-reviewed journal, but there is no plan to specifically disseminate the findings to study participants.

\section{DISCUSSION}

This is the first study the authors are aware of to measure health assets in hospitalised older adults in a systematic way. Health assets have been shown to play an important role in mitigating the effects of frailty for older adults in the community setting. The cumulative effect of this has not been explored systematically in the hospital setting.

Mortality and functional decline have been chosen as the outcomes of interest as hospital is a time of excess risk for these outcomes. ${ }^{812}$ The development of new disability is highly prevalent in hospitalised older adults, particularly for those who are frail. ${ }^{13}$ The prognosis for this is poor, and of those who leave hospital with a new disability at 1 year, $41 \%$ have died and only $30 \%$ have recovered to their previous functional state. ${ }^{9}$

The ability of an individual to recover from an acute illness and to return to their home environment is dependent on factors additional to the acute illness. Individual resources, such as social supports, ${ }^{14}$ adequate financial resources ${ }^{15}$ and the ability to access and emotional support, ${ }^{16}$ have been demonstrated to be protective. Social vulnerability and socioeconomic factors are linked with frailty. ${ }^{1718}$ This study will help clarify whether a higher 
level of health assets mitigate frailty associated outcomes or if their impact is primarily related to contribution to baseline frailty in this setting.

The secondary outcomes of increased length of stay and readmission have been chosen as in a systematic review health assets impacted these outcomes.

Health assets can be measured at any time during the hospital admission. Most of the health assets, such as those measuring education, family and financial resources, will not alter depending on time of measurement. The frailty index has predictive validity whether it is measured at the time of admission or later in the hospital admission. ${ }^{419}$

A limitation of the study is that not all patients admitted during the time period will be able to be approached. Increasing frailty is associated with increased length of stay. ${ }^{419}$ Length of stay is also impacted by the community resources available in individual health services, which limits generalisability. Readmission is not associated with frailty status but is predicted by social vulnerability, ${ }^{20}$ so the HAI may be predictive for this outcome.

A strength of the methodology is the utilisation of significant amounts of routine data. This decreases the burden for participants in participating. The inclusion of participants with cognitive impairment by obtaining consent from a next of kin will also improve the generalisability.

Despite attempting to identify all health assets with a systematic review as well as interrogation of the interRAI database, ${ }^{3}$ it is possible that not all health assets were identified. It also remains to be seen whether health assets are specific to a sociocultural setting.

Health assets can lead to improved outcomes for hospitalised older adults. Validation of the HAI will enable better risk stratification. Understanding of factors that mitigate the effects of frailty could also lead to the development of interventions to facilitate recovery following admission to hospital.

Contributors All authors contributed significantly to the development of this manuscript. KJG contributed to design of the protocol and preparation of the manuscript. REH, NMP and WKL contributed to design of the protocol and revision of the manuscript. All authors gave final approval to the manuscript.

Funding This research received no specific grant from any funding agency in the public, commercial or not-for-profit sectors. This study will form part of a PhD being completed by KJG. KJG is supported by scholarships from the Australian Postgraduate Association through the University of Melbourne and the Northern Health Foundation.

Competing interests None declared.

Patient consent Not required.

Ethics approval Ethical approval has been obtained from Austin Health high-risk ethics committee.

Provenance and peer review Not commissioned; externally peer reviewed.
Open Access This is an Open Access article distributed in accordance with the Creative Commons Attribution Non Commercial (CC BY-NC 4.0) license, which permits others to distribute, remix, adapt, build upon this work non-commercially, and license their derivative works on different terms, provided the original work is properly cited and the use is non-commercial. See: http://creativecommons.org/ licenses/by-nc/4.0/

(c) Article author(s) (or their employer(s) unless otherwise stated in the text of the article) 2018. All rights reserved. No commercial use is permitted unless otherwise expressly granted.

\section{REFERENCES}

1. Gregorevic KJ, Lim WK, Peel NM, et al. Are health assets associated with improved outcomes for hospitalised older adults? A systematic review. Arch Gerontol Geriatr 2016;67:14-20.

2. Lindström B, Eriksson M. Salutogenesis. J Epidemiol Community Health 2005;59:440-2.

3. Hubbard RE, Peel NM, Samanta M, et al. Derivation of a frailty index from the interRAl acute care instrument. BMC Geriatr 2015;15:27.

4. Evans SJ, Sayers M, Mitnitski A, et al. The risk of adverse outcomes in hospitalized older patients in relation to a frailty index based on a comprehensive geriatric assessment. Age Ageing 2014;43:127-32.

5. Hubbard RE, Peel NM, Samanta M, et al. Frailty status at admission to hospital predicts multiple adverse outcomes. Age Ageing 2017;46:801-6.

6. Searle SD, Mitnitski A, Gahbauer EA, et al. A standard procedure for creating a frailty index. BMC Geriatr 2008;8:24.

7. Wang C, Song X, Mitnitski A, et al. Effect of health protective factors on health deficit accumulation and mortality risk in older adults in the Beijing Longitudinal Study of Aging. J Am Geriatr Soc 2014;62:821-8.

8. Covinsky KE, Pierluissi E, Johnston CB. Hospitalization-associated disability: "She was probably able to ambulate, but I'm not sure". JAMA 2011;306:1782-93.

9. Boyd CM, Landefeld CS, Counsell SR, et al. Recovery of activities of daily living in older adults after hospitalization for acute medical illness. J Am Geriatr Soc 2008;56:2171-9.

10. Collins GS, Reitsma JB, Altman DG, et al. Transparent reporting of a multivariable prediction model for individual prognosis or diagnosis (TRIPOD): the TRIPOD statement. BMJ 2015;350:g7594.

11. Subbe CP, Kruger M, Rutherford P, et al. Validation of a modified Early Warning Score in medical admissions. QJM 2001;94:521-6.

12. Hoogerduijn JG, Buurman BM, Korevaar JC, et al. The prediction of functional decline in older hospitalised patients. Age Ageing 2012;41:381-7

13. Gill TM, Allore HG, Gahbauer EA, et al. Change in disability after hospitalization or restricted activity in older persons. JAMA 2010;304:1919-28

14. Dent E, Hoogendijk EO. Psychosocial factors modify the association of frailty with adverse outcomes: a prospective study of hospitalised older people. BMC Geriatr 2014;14:108.

15. Li AK, Covinsky KE, Sands LP, et al. Reports of financial disability predict functional decline and death in older patients discharged from the hospital. J Gen Intern Med 2005;20:168-74.

16. Berkman LF, Leo-Summers L, Horwitz RI. Emotional support and survival after myocardial infarction. A prospective, population-based study of the elderly. Ann Intern Med 1992;117:1003-9.

17. Andrew MK, Mitnitski AB, Rockwood K. Social vulnerability, frailty and mortality in elderly people. PLoS One 2008;3:e2232.

18. Marshall A, Nazroo J, Tampubolon G, et al. Cohort differences in the levels and trajectories of frailty among older people in England. $J$ Epidemiol Community Health 2015;69:316-21.

19. Krishnan M, Beck S, Havelock W, et al. Predicting outcome after hip fracture: using a frailty index to integrate comprehensive geriatric assessment results. Age Ageing 2014;43:122-6.

20. Cooksley T, Nanayakkara PW, Nickel CH, et al. Readmissions of medical patients: an external validation of two existing prediction scores. QJM 2016;109:245-8. 